\title{
The 4-hydroxynonenal mediated oxidative damage of blood proteins and lipids involves secondary lipid peroxidation reactions
}

\author{
AYMAN G. MUSTAFA ${ }^{1,2}$, MAHMOUD A. ALFAQIH ${ }^{3}$ and OTHMAN AL-SHBOUL ${ }^{3}$ \\ ${ }^{1}$ Department of Basic Medical Sciences, School of Medicine, Jordan University of Science and Technology, Irbid 22110, \\ Jordan; ${ }^{2}$ Department of Anatomy, School of Medicine, Qatar University, Doha, Qatar; ${ }^{3}$ Department of Physiology \\ and Biochemistry, School of Medicine, Jordan University of Science and Technology, Irbid 22110, Jordan
}

Received November 30, 2017; Accepted May 25, 2018

DOI: $10.3892 / e t m .2018 .6419$

\begin{abstract}
Lipid peroxidation is associated with several metabolic diseases. Lipid peroxidation causes cellular damage through reactive aldehyde species such as 4-hydroxyonenal (4-HNE). The exact mechanism(s) by which 4-HNE causes damage in the intravascular compartment is not yet exactly understood. Using an in vitro system, the damage induced by 4-HNE on the blood was investigated by measuring protein carbonyl groups and thiobarbituric acid reactive substances (TBARS) following 4-HNE treatment. The findings demonstrated that treatment with 4-HNE increased the carbonylation of protein and the formation of TBARS in the blood plasma. It was also tested whether phenelzine, a scavenger of aldehyde species, or U-83836E, a scavenger of lipid peroxy radicals, attenuated the damage caused by 4-HNE. It was demonstrated that phenelzine or U-83836E both mitigated the effects of 4-HNE on the proteins and the lipids of the blood plasma. The findings of the current study suggest that phenelzine, U-83836E or functionally similar therapeutics may prevent or treat diseases that involve an increased production of 4-HNE in the intravascular compartment.
\end{abstract}

\section{Introduction}

Reactive oxygen species (ROS) are considered to be normal by-products of cellular respiration and inflammation (1), nonetheless, extensive evidence has accumulated over the last four decades that links the excessive production of ROS with cellular pathology and tissue damage (1-4). Cells exhibit several mechanisms to antagonize the cytotoxic effects of ROS, but these mechanisms can be overwhelmed, producing

Correspondence to: Dr Mahmoud A. Alfaqih, Department of Physiology and Biochemistry, School of Medicine, Jordan University of Science and Technology, Health Sciences Complex, M3L0, Irbid 22110, Jordan

E-mail: maalfaqih@just.edu.jo

Key words: thiobarbituric acid reactive substances, reactive oxygen species, oxidative stress, phenelzine, lipid peroxidation what is known as 'oxidative stress' (5), which is a state that contributes to the pathogenesis of a number of chronic disease conditions including Alzheimer's disease (6,7), traumatic brain injury $(8)$, diabetes $(9,10)$ and ageing $(11)$.

The well-established role ROS serves in disease etiology has provided rationale for research aiming to elucidate the exact mechanism(s) by which ROS can initiate and/or propagate cellular pathology (12-14). Although this remains to be completely understood, there is evidence which indicates that ROS can produce cellular damage by: i) Peroxidation of polyunsaturated fatty acids (PUFA) of cellular membranes (15), ii) inducing mutations through the nitration and deamination of DNA $(16,17)$, and iii) disrupting cellular function through protein nitration and carbonylation (18). The above effects may be triggered by ROS directly or through the decomposition of ROS into other highly reactive and cell damaging radicals (1).

Lipid peroxidation refers to the process by which the hydrogen atom of the double bonds of PUFAs is attacked by oxygen radicals (primarily the hydroxyl and hydroperoxyl radicals) $(19,20)$. The consequence of this attack is the formation of a lipid radical, which can directly react with oxygen to form a lipid peroxyl radical (20). The lipid peroxyl radical formed from the above reaction is highly reactive and can remove another hydrogen from the double bond of a neighboring PUFA, resulting in a chain reaction (20). The above self-propagating chain of events may be terminated by natural antioxidants like vitamin $\mathrm{E}$ that can donate protons to lipid peroxyl radicals thereby forming lipid hydroperoxides (21). The resulting vitamin $\mathrm{E}$ free radical can react with yet another lipid peroxyl radical to form non-radical species. Lipid peroxyl radicals formed in this way can also cyclize into cyclic peroxide species and finally be converted into secondary aldehyde species like malondialdehyde (MDA) and 4-hydroxynonenal (4-HNE) (22).

The detrimental effects caused by lipid peroxidation can be attributed to the loss of membrane integrity described above with the resulting lack of control over ion trafficking and cellular osmolality (23), or cytotoxic and mutagenic properties of the secondary aldehyde species (MDA and 4-HNE) that result from lipid peroxidation chain reactions (23). In particular, the role of 4-HNE has received considerable interest in recent years and has been the target of extensive research $(19,24,25)$. One mechanism by which 4 -HNE produces cell toxicity is 
believed to involve the reaction of 4-HNE with the thiol and amino groups of macromolecules (19). Another mechanism involves a potential role that 4-HNE serves as a secondary messenger of lipid peroxidation where it modulates gene expression through regulating the effect of a number of transcription factors $(26,27)$. Nonetheless, the exact mechanism by which 4-HNE produces its effect is not yet completely understood.

Using blood plasma and isolated platelets as a model system, the present authors previously performed an in vitro assay that recapitulated the effects of peroxynitrite, a reactive nitrogen species, on protein oxidation and lipid peroxidation (13). Notably, it was demonstrated that using drugs that catalytically decompose peroxynitrite-derived free radicals (nitrogen dioxide, carbonate and superoxide) mitigates the oxidative damage mediated by peroxynitrite treatment (13). In the present study, using the in vitro system described above, it was hypothesized that by using different antioxidant agents that target different steps of the lipid peroxidation reactions triggered by 4-HNE, the mechanism by which $4-\mathrm{HNE}$ produces oxidative damage may be elucidated. The present study reports the protective effects of U-83836E (a scavenger of lipid peroxyl radicals) and phenelzine (a scavenger of reactive aldehyde species) against the 4-HNE-mediated oxidative damage of blood proteins and lipids. The mechanism by which 4-HNE induces oxidative damage is discussed in the context of the above observations.

\section{Materials and methods}

Subject characteristics and blood sample collection. The present prospective study was approved by the Institutional Review Board affiliated with the Jordan University of Science and Technology (JUST; Irbid, Jordan).

Recruitment of study participants took place at the Family Medicine Clinics of King Abdullah University Hospital (KAUH). KAUH is a tertiary hospital affiliated with JUST. Study participants were initially interviewed with a clinical research coordinator who explained the research and its objectives. Relevant medical history was collected into a structured data collection sheet by the research coordinator. To be eligible to participate in the study, the study participants needed to be of Jordanian descent and 22-25 years of age. Individuals who were; i) habitual cigarette or water pipe smokers, ii) suffered from hypertension, type 1 or type 2 diabetes mellitus, atherosclerosis or dyslipidemia as indicated by their medical history; or iii) received anti-histamines or nonsteroidal anti-inflammatory drugs up $\leq 2$ weeks prior to the interview were excluded from participating in the study. Following the application of the eligibility criteria explained above, 2 male and 1 female subjects consented to participate and were thus recruited to the study.

Following providing informed written consent, $500 \mathrm{ml}$ blood was collected from the subject by a certified phlebotomist into a $250 \mathrm{ml}$ Citrate-Phosphate-Dextrose with Adenine (CPDA1) bag. The blood was then divided into ten individual CPDA1 tubes for the purpose of separating the blood plasma and platelets, and to receive different treatments.

Isolation of plasma and platelets from whole blood. Plasma and platelets were isolated as previously described (13). Briefly, whole blood was centrifuged at $450 \mathrm{x}$ g for $6.20 \mathrm{~min}$ at room temperature to recover platelet-rich plasma. The platelet-rich plasma recovered from the previous step was then subjected to centrifugation at 2,500 x g for $5.40 \mathrm{~min}$ at room temperature; leading to sedimentation of a platelet-rich pellet. The pellet recovered from the previous step was then washed twice with Tyrode's buffer (Sigma-Aldrich; Merck KGaA, Darmstadt, Germany) and re-suspended in the same buffer. The final platelet number was measured with a hemocytometer and accordingly adjusted to $10^{9}$ platelets $/ \mathrm{ml}$ suspensions.

Sample treatment. The experimental design and sample treatments were previously described (13). Briefly, the plasma and isolated platelets were randomly divided into 6-well plates and treated with either vehicle (DMSO) or an increasing dose of 4-HNE $(0.1,1$ or $10 \mu \mathrm{M})$. In another 6-well plate, samples were treated with a combination of $4-\mathrm{HNE}(10 \mu \mathrm{M})$ with the following doses of either phenelzine $(25,50$ or $100 \mu \mathrm{M}$; MP Biochemicals, LLC, Santa Ana, CA, USA) or U-83836E (25, 50 or $100 \mu \mathrm{M}$; Biomol International; Enzo Life Sciences, Inc., Farmingdale, NY, USA).

Estimation of total plasma proteins. The total protein content of plasma samples was estimated using a Pierce ${ }^{\mathrm{TM}}$ BCA Protein Assay kit (cat. no. 23225; Thermo Fisher scientific, Inc., Waltham, MA, USA) according to the manufacturer's protocol. Absorbance was determined at $562 \mathrm{~nm}$ on an ELx800 ELISA reader (BioTek Instruments, Inc., Winooski, VT, USA).

Detection of carbonyl group and thiobarbituric acid reactive substances (TBARS). The methods used for the detection of carbonyl groups and TBARS were previously detailed (13). Final measurement of the content of carbonyl groups in each sample was performed using a Protein Carbonyl Content Assay kit (cat. no. ab126287; Abcam, Cambridge, UK). Absorbance was measured at $375 \mathrm{~nm}$ on an ELx800 ELISA reader (BioTek Instruments, Inc., Winooski, VT, USA). Final measurement of TBARS concentration was performed spectrophotometrically using a Lipid Peroxidation Assay kit (cat. no. MAK085; Sigma-Aldrich; Merck KGaA). Absorbance was measured at $530 \mathrm{~nm}$ on an ELx800 ELISA reader.

Statistical analysis. Statistical analysis was performed using SPSS version 17 (SPSS, Inc., Chicago, IL, USA). Data were expressed as the mean \pm standard deviation. A one-way analysis of variance was used to compare data in the different experimental groups followed by Fisher's post hoc test. $\mathrm{P}<0.05$ was considered to indicate statistically significant differences.

\section{Results}

The concentration of protein carbonyl groups and TBARS in blood plasma and platelets is increased by 4-HNE. In a previous study, the present authors described an in vitro assay that allowed investigation of the oxidative damage induced by the reactive nitrogen species peroxynitrite in blood lipids and proteins (12). Prior to attempting to understand the mechanism of 4-HNE-induced oxidative damage in the present study, the aim was to quantitatively evaluate the oxidative damage induced by 4-HNE using well-established assays. To achieve 


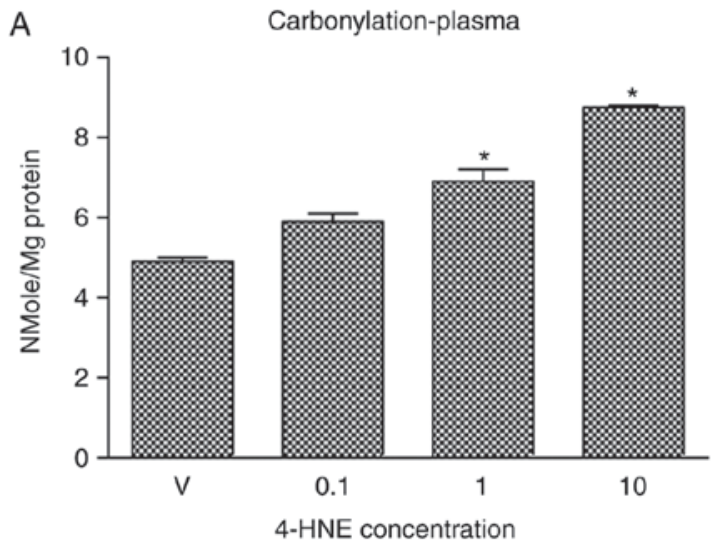

B

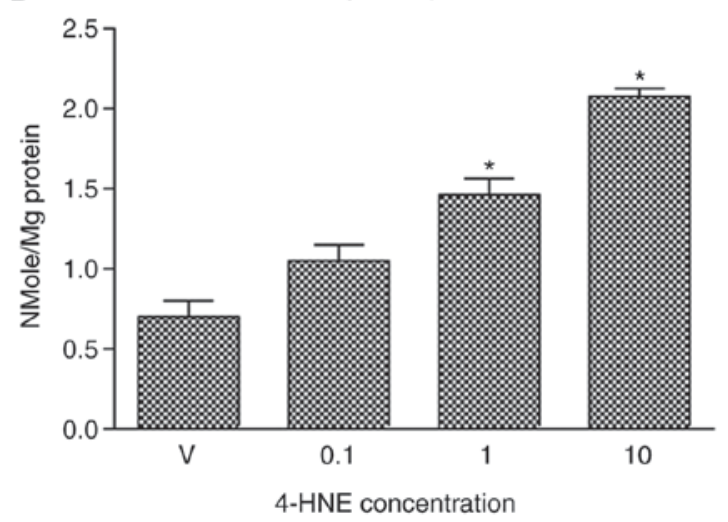

Figure 1. Treatment with 4-HNE induces carbonyl group formation in plasma proteins and platelets. (A) Plasma and (B) platelets were treated with vehicle control or 4-HNE $(0.1,1$ or $10 \mu \mathrm{M})$ at room temperature for $20 \mathrm{~min}$. The samples were then collected, and protein carbonyl formation was measured using a colorimetric based method. The results are expressed as nMole of protein carbonyl group formation per mg of total protein. The results are representative of three independent experiments performed in triplicate. Data are expressed as the mean \pm standard deviation. "P<0.05 vs. V. 4-HNE, 4-hydroxyonenal; V, vehicle control group.

this goal, blood plasma and platelet samples were treated with 4-HNE $(0.1,1$ or $10 \mu \mathrm{M})$ and the concentration of carbonyl group formation (a biomarker of protein oxidative damage) and TBARS (a biomarker of lipid peroxidative damage) induced by 4-HNE treatment was then measured. It was indicated that treating blood plasma (Fig. 1A) or platelets (Fig. 1B) with 1 or $10 \mu \mathrm{M} 4$-HNE significantly increased carbonyl group concentration. Likewise, treatment of blood plasma (Fig. 2A) or platelets (Fig. 2B) with 1 or $10 \mu \mathrm{M} 4$-HNE significantly increased TBARS concentration. Treatment with $0.1 \mu \mathrm{M}$ 4-HNE induced a significant increase in TBARS concentration in platelets, but not in blood plasma samples.

Phenelzine antagonizes the effect of 4-HNE on blood plasma and platelet proteins and lipids. Following quantitatively measuring the oxidative damage induced by 4-HNE at two end points (protein carbonyl and TBARS formation), the aim was to elucidate the mechanism by which 4-HNE produces these effects. Phenelzine is a drug that contains a hydrazine moiety that allows it to function as an aldehyde scavenger (28), and 4-HNE is an aldehyde free radical species that results from lipid peroxidation (29). It was therefore hypothesized that phenelzine could reverse the oxidative damage produced
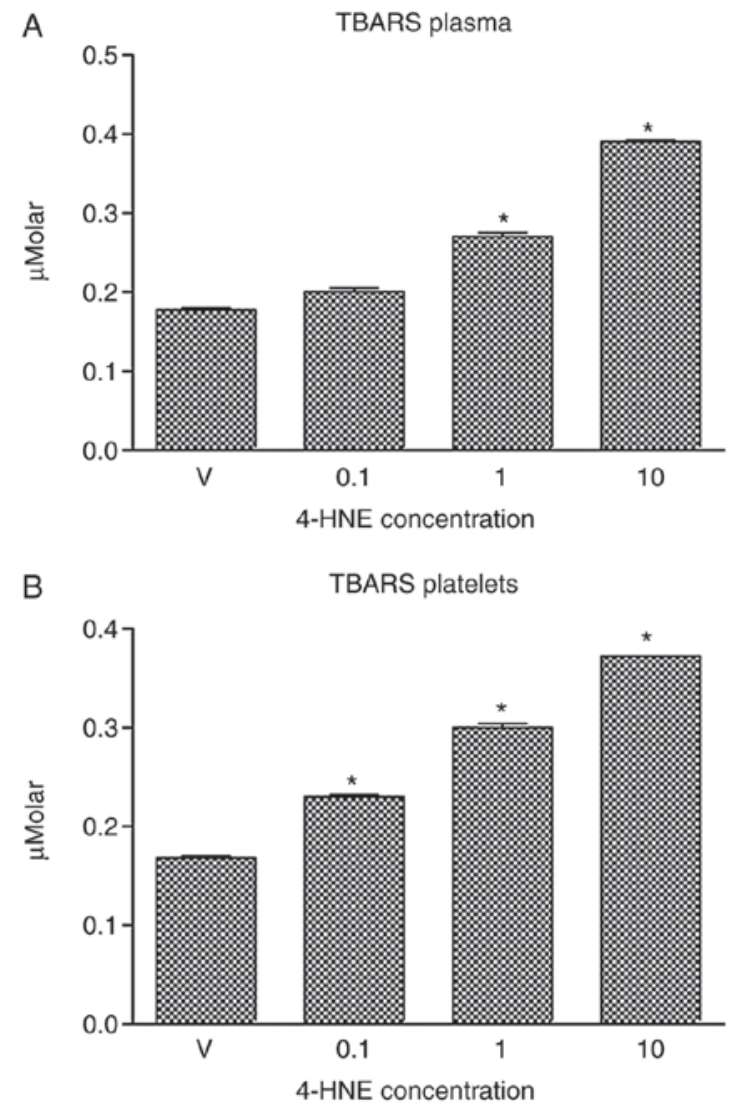

Figure 2. 4-HNE treatment induces TBARS formation in plasma proteins and platelets. (A) Plasma or (B) platelets were treated with vehicle control or 4-HNE $(0.1,1$ or $10 \mu \mathrm{M})$. All treatments were performed at room temperature for $20 \mathrm{~min}$. The samples were subsequently collected, and the formation of (TBARS) was measured using a colorimetric based assay. The results are expressed as $\mu \mathrm{M}$ concentration of TBARS and are representative of three independent experiments performed in triplicate. Data are expressed as the mean \pm standard deviation. ${ }^{*} \mathrm{P}<0.05$ vs. V. 4-HNE, 4-hydroxyonenal; TBARS, thiobarbituric acid reactive substances; V, vehicle control group.

by 4-HNE if these effects were associated with its chemical character as a free aldehyde radical. As such, plasma and platelet samples were treated with 4-HNE accompanied with an increasing dose of phenelzine, and protein carbonyl and TBARS formation was measured in the treated samples. Notably, phenelzine partially reversed the protein carbonyl (Fig. 3A) and TBARS (Fig. 3B) formation produced by 4-HNE treatment in blood plasma and platelet samples. These findings suggest that the oxidative damage caused by 4-HNE is at least partially due to its aldehyde free radical character.

\section{U-83836E antagonizes the effect of 4-HNE on blood plasma} and platelet proteins and lipids. Lipid peroxidation proceeds through the formation of a lipid radical followed by the generation of a lipid peroxyl radical. If the oxidative damage caused by 4-HNE treatment depends on initiating secondary lipid peroxidative damage, it may be concluded that limiting the concentration of lipid peroxyl radicals would reduce the oxidative damage triggered by 4-HNE. To explore this, plasma and platelet samples were treated with 4-HNE alone or in combination with the lipid peroxyl radical scavenger, U-83836E (30), and protein carbonylation and TBARS formation were estimated. Treatment with U-83836E antagonized 
A

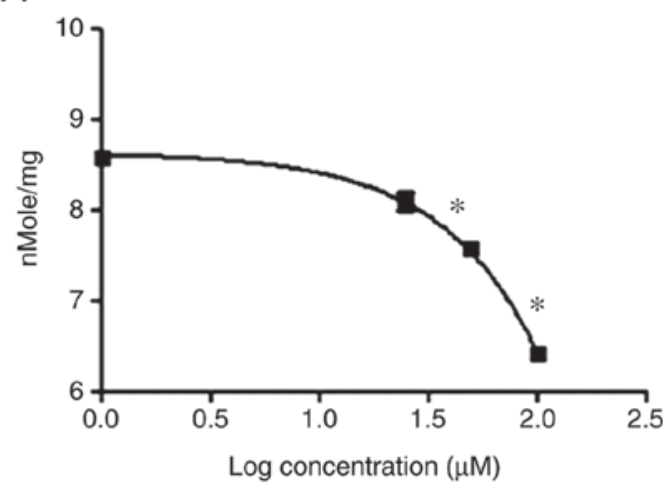

B

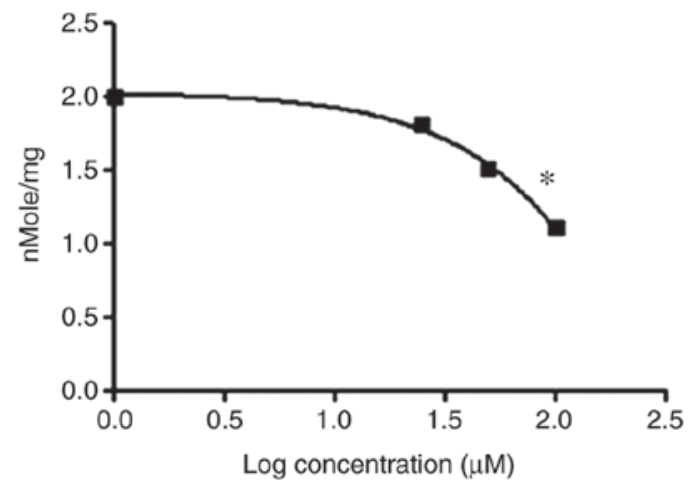

TBARS-plasma

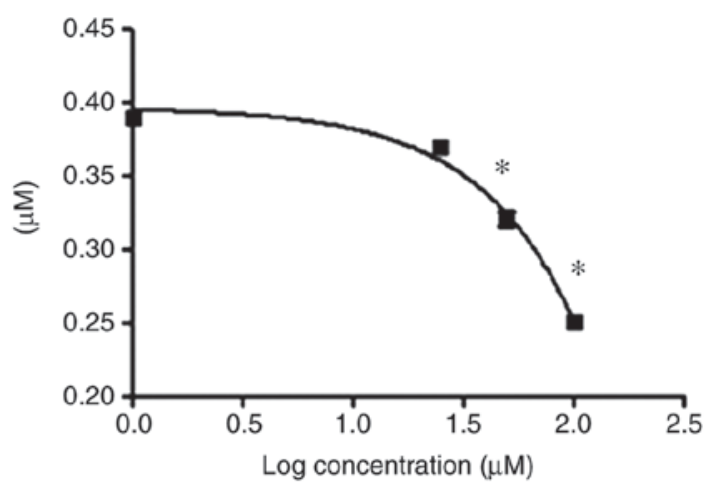

TBARS-platelets

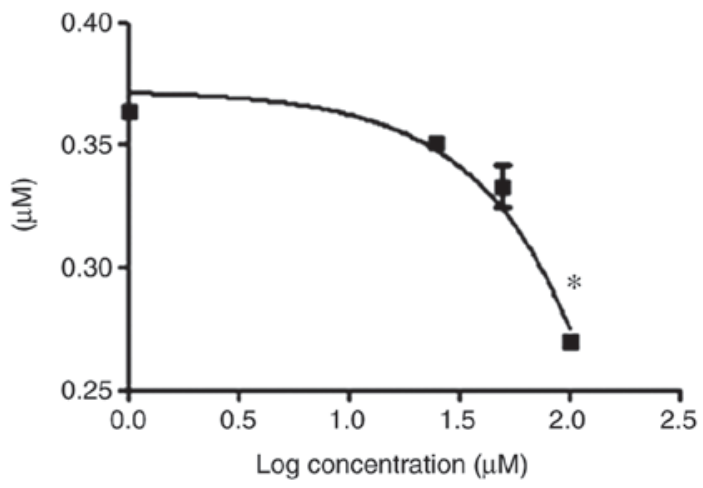

Figure 3. Phenelzine reduces the ability of 4-HNE to induce protein carbonyl and TBARS formation in plasma and platelet proteins and lipids. (A) Plasma and (B) platelet samples were treated with $10 \mu \mathrm{M} 4$-HNE alone or in combination with phenelzine $(25,50$ or $100 \mu \mathrm{M})$. Protein carbonylation or TBARS formation was later measured using colorimetric based assays. The results are representative of three independent experiments performed in triplicate. Data are expressed as the mean \pm standard deviation. ${ }^{*} \mathrm{P}<0.05$ vs. the control group $(0 \mu \mathrm{M}$ phenelzine $)$. 4-HNE, 4-hydroxyonenal; TBARS, thiobarbituric acid reactive substances.

the effects of 4-HNE on protein carbonylation and TBARS formation in blood plasma (Fig. 4A) and platelet (Fig. 4B) samples. These results suggest that the oxidative damage induced by 4-HNE treatment is associated with the formation of lipid peroxyl radicals; radicals known to be produced in lipid peroxidation reactions.

\section{Discussion}

The findings of the present study provide a mechanistic link between a reactive aldehyde species (4-HNE) produced from lipid peroxidation of biological membranes and increased oxidative stress in the intravascular compartment. The results also highlight several pathways that may be pharmacologically targeted to antagonize the impact of 4-HNE production. Furthermore, the correct interpretation of these results aids in the understanding of the exact mechanism(s) by which 4-HNE produces its pathophysiological effects whether in the intravascular compartment or in other biological niches. Pharmacological mitigation of the effects of 4-HNE may be useful for the therapeutic intervention of several chronic diseases such as traumatic brain injury, Alzheimer's disease and diabetes.

Notable in the present study was the observation that 4-HNE, considered an end product of lipid peroxidation, leads to secondary lipid peroxidative damage as evidenced by an increase in TBARS formation (a biomarker of lipid peroxidation) in blood plasma and isolated platelets treated directly with 4-HNE. A direct causal relationship between increased 4-HNE concentration and the peroxidative damage of membrane lipids is supported by the ability to reverse TBARS formation induced by 4-HNE treatment via the use of a scavenger of reactive aldehyde species, phenelzine. As 4-HNE is a reactive aldehyde species, phenelzine will presumably reduce the concentration of 4-HNE available to induce the peroxidation of biological membranes. In another experiment, TBARS formation induced by 4-HNE treatment was reduced via the use of the lipid peroxyl radical chelator, U-83836E. Lipid peroxyl radicals are produced during lipid peroxidation following the reaction of oxygen with lipid radicals, which are themselves produced as a result of the abstraction of a proton from the double bonds of PUFAs of biological membranes by oxygen radicals (hydroxyl and hydroperoxyl radicals). As 4-HNE is not an oxygen radical itself, it is unlikely to directly cause the formation of lipid radicals. However, it seems that an increase in the concentration of 4-HNE can indirectly cause an increase in the concentration of oxygen radicals, which may initiate a new cycle of lipid peroxidation. In accordance with the above tentative series of events is the finding that 4-HNE can deplete the sulfhydryl groups of glutathione (31), which would presumably increase the concentration of hydrogen peroxide, which can in turn decompose to hydroxyl radicals.

In addition to demonstrating that 4-HNE direct treatment causes secondary peroxidative damage of blood and platelet lipids, 
A

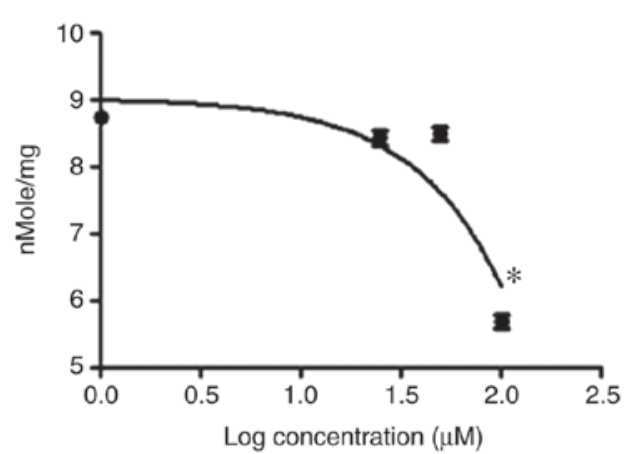

B

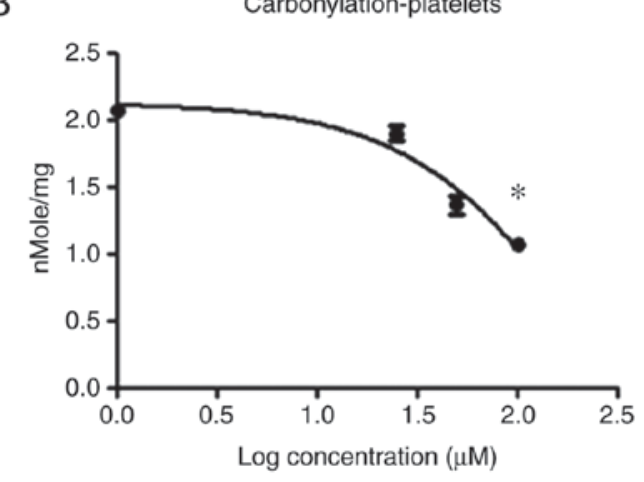

TBARS-plasma

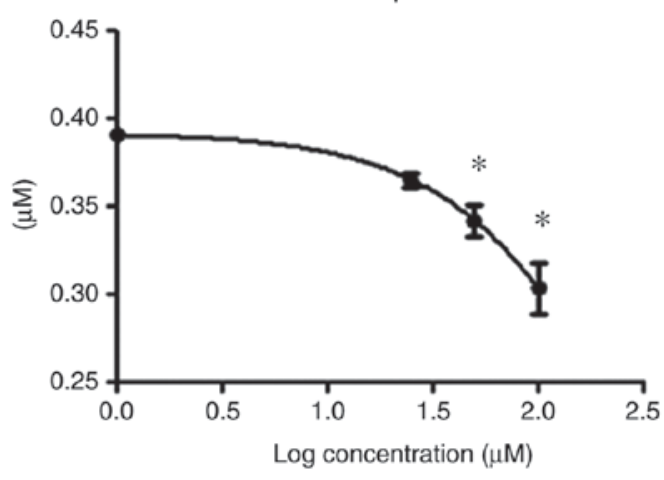

TBARS-platelets

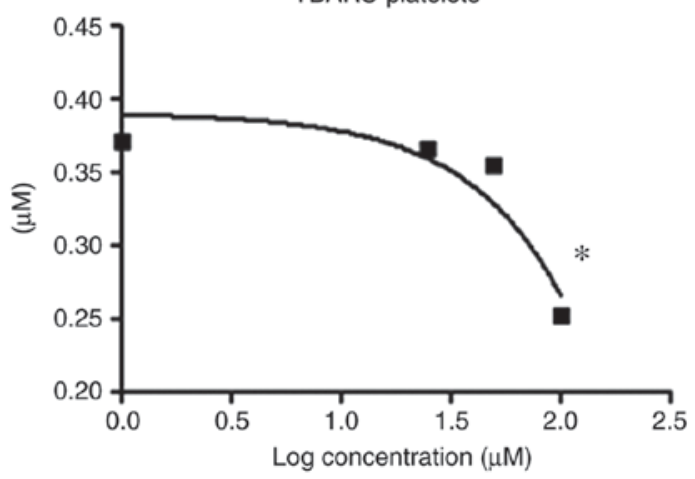

Figure 4. U-83836E reduces the ability of 4-HNE to induce protein carbonyl and TBARS formation in plasma proteins and platelets. (A) Plasma and (B) platelet samples were treated with $10 \mu \mathrm{M}$ 4-HNE alone or in combination with $\mathrm{U}-83836 \mathrm{E}(25,50$ or $100 \mu \mathrm{M})$. Protein carbonylation or TBARS formation was then measured using colorimetric based assays. The results are representative of three independent experiments performed in triplicate. Data are expressed as the mean \pm standard deviation. "P<0.05 vs. the control group $(0 \mu \mathrm{M}$ U-83836E). 4-HNE, 4-hydroxyonenal; TBARS, thiobarbituric acid reactive substances.

it was also demonstrated that 4-HNE induced oxidative damage of blood and platelet proteins in the form of increased protein carbonylation. This is not surprising as it is well documented that electrophiles such as 4-HNE can react in Michael addition with side chains of cysteine and histidine and cause increased protein carbonylation (32). The direct role of 4-HNE in increasing protein carbonylation can be further validated through the present finding that reducing the effective concentration of 4-HNE in via chelating it with phenelzine decreased protein carbonylation. It was also demonstrated that interrupting the cycle of lipid peroxidation by scavenging lipid peroxyl radicals with U-83836E reduced the effect of 4-HNE on protein carbonylation. These findings suggest that 4-HNE treatment can induce secondary lipid peroxidation reactions, which can lead to the formation of increasing amounts of 4-HNE (and other reactive aldehyde species), which may cause yet more protein carbonylation.

In addition to the role of 4-HNE in inducing its oxidative damage on lipids and proteins in the context of secondary lipid peroxidation reactions; several observations indicate that 4-HNE can also act as a cell-signaling molecule (33). Alteration of cell signaling pathways, including the mitogen-activated protein kinase (34) and nuclear factor (erythroid-derived 2)-like 2 (35) pathways, may contribute to the mechanisms which allow HNE to mediate oxidative damage, or they may have their own independent effect. The role of these pathways in 4-HNE mediated oxidative damage is an active area of research for the present authors.

In conclusion, the present findings demonstrated that 4-HNE treatment increased the rate of peroxidation of lipids, and the oxidative damage of proteins in the blood and in isolated platelets. The data indicates that this may be mitigated by chelating 4-HNE, or any other reactive aldehyde species, with phenelzine or by scavenging lipid peroxyl radicals with $\mathrm{U}-83836 \mathrm{E}$. The data presented is in accordance with a model in which 4-HNE is associated a chain reaction that results in secondary lipid peroxidation and progressively increasing amounts of 4-HNE. Based on these results, it appears that the therapeutic options to mitigate the cytotoxic effects of 4-HNE in the intravascular compartment should involve approaches that reduce 4-HNE itself, interrupt the lipid peroxidation process, or involve both methods in combination.

\section{Acknowledgements}

Not applicable.

\section{Funding}

The present study was supported by the Deanship of Research at Jordan University of Science and Technology (grant no. 301/2014).

\section{Availability of data and materials}

The datasets generated and/or analyzed during the current study are available from the corresponding author on reasonable request. 


\section{Authors' contributions}

All the authors participated in the design of the study, analysis of the data and final review of the manuscript. AGM and OA-S conceived the study and performed all the biochemical measurements. AGM, MAA and OA-S collected the data. MAA performed the statistical analysis. MAA and AGM drafted the manuscript. All authors read and approved the final manuscript.

\section{Ethics approval and consent to participate}

The present study was approved by the Institutional Review Board affiliated with the Jordan University of Science and Technology (Irbid, Jordan). All participants provided written informed consent.

\section{Patient consent for publication}

All participants provided written informed consent.

\section{Competing interests}

The authors declare that they have no competing interests.

\section{References}

1. Ray PD, Huang BW and Tsuji Y: Reactive oxygen species (ROS) homeostasis and redox regulation in cellular signaling. Cell Signal 24: 981-990, 2012.

2. Bergamini CM, Gambetti S, Dondi A and Cervellati C: Oxygen, reactive oxygen species and tissue damage. Curr Pharm Des 10: 1611-1626, 2004

3. Bandyopadhyay U, Das D and Banerjee RK: Reactive oxygen species: Oxidative damage and pathogenesis. Current science: 658-666, 1999

4. Halliwell B: Reactive oxygen species in living systems: Source, biochemistry, and role in human disease. Am J Med 91: S14-S22, 1991.

5. Betteridge DJ: What is oxidative stress? Metabolism 49 (2 Suppl 1): 3-8, 2000.

6. Shukla V, Mishra SK and Pant HC: Oxidative stress in neurodegeneration. Adv Pharmacol Sci 2011: 572634, 2011.

7. Andersen JK: Oxidative stress in neurodegeneration: Cause or consequence? Nat Med 10 (Suppl): S18-S25, 2004.

8. Rodriguez-Rodriguez A, Egea-Guerrero JJ, Murillo-Cabezas F and Carrillo-Vico A: Oxidative stress in traumatic brain injury. Current Med Chem 21: 1201-1211, 2014.

9. Giacco F and Brownlee M: Oxidative stress and diabetic complications. Circ Res 107: 1058-1070, 2010.

10. Asmat U, Abad K and Ismail K: Diabetes mellitus and oxidative stress-A concise review. Saudi Pharm J 24: 547-553, 2016.

11. Liochev SI: Reactive oxygen species and the free radical theory of aging. Free Radic Biol Med 60: 1-4, 2013.

12. Mustafa AG, Al-Shboul O, Alfaqih MA, Al-Qudah MA and Al-Dwairi AN: Phenelzine reduces the oxidative damage induced by peroxynitrite in plasma lipids and proteins. Arch Physiol Biochem: 1-6, 2017.

13. Mustafa AG, Bani-Ahmad MA, Jaradat AQ and Allouh MZ: Tempol protects blood proteins and lipids against peroxynitrite-mediated oxidative damage. Exp Biol Med (Maywood) 240: 109-112, 2015.

14. Mustafa AG, Singh IN, Wang J, Carrico KM and Hall ED: Mitochondrial protection after traumatic brain injury by scavenging lipid peroxyl radicals. J Neurochem 114: 271-280, 2010.
15. Mylonas C and Kouretas D: Lipid peroxidation and tissue damage. In vivo 13: 295-309, 1999.

16. Jena NR: DNA damage by reactive species: Mechanisms, mutation and repair. J Biosci 37: 503-517, 2012.

17. Hemnani T and Parihar MS: Reactive oxygen species and oxidative DNA damage. Indian J Physiol Pharmacol 42: 440-452, 1998.

18. Suzuki YJ, Carini M and Butterfield DA: Protein Carbonylation. Antioxid Redox Signal 12: 323-325, 2010.

19. Ayala A, Muñoz MF and Argüelles S: Lipid peroxidation: Production, metabolism, and signaling mechanisms of malondialdehyde and 4-Hydroxy-2-Nonenal. Oxid Med Cell Longev 2014: 360438, 2014.

20. Yin $\mathrm{H}, \mathrm{Xu} \mathrm{L}$ and Porter NA: Free radical lipid peroxidation: Mechanisms and analysis. Chem Rev 111: 5944-5972, 2011.

21. Girotti AW: Lipid hydroperoxide generation, turnover, and effector action in biological systems. J Lipid Res 39: 1529-1542, 1998.

22. Reis A and Spickett CM: Chemistry of phospholipid oxidation. Biochim Biophys Acta 1818: 2374-2387, 2012.

23. Dix TA and Aikens J: Mechanisms and biological relevance of lipid peroxidation initiation. Chem Res Toxicol 6: 2-18, 1993.

24. Zhong $\mathrm{H}$ and $\mathrm{Yin} \mathrm{H}$ : Role of lipid peroxidation derived 4-hydroxynonenal (4-HNE) in cancer: Focusing on mitochondria. Redox Biol 4: 193-199, 2015.

25. Schaur RJ, Siems W, Bresgen N and Eckl PM: 4-Hydroxy-nonenal-A bioactive lipid peroxidation product. Biomolecules 5: 2247-2337, 2015.

26. Yang Y, Sharma R, Sharma A, Awasthi S and Awasthi YC: Lipid peroxidation and cell cycle signaling: 4-hydroxynonenal, a key molecule in stress mediated signaling. Acta Biochim Pol 50: 319-336, 2003.

27. Jang EJ, Jeong HO, Park D, Kim DH, Choi YJ, Chung KW, Park MH, Yu BP and Chung HY: Src tyrosine kinase activation by 4-hydroxynonenal upregulates p38, ERK/AP-1 signaling and COX-2 expression in YPEN-1 Cells. PLoS One 10: e0129244, 2015.

28. Cebak JE, Singh IN, Hill RL, Wang JA and Hall ED: Phenelzine protects brain mitochondrial function in vitro and in vivo following traumatic brain injury by scavenging the reactive carbonyls 4-hydroxynonenal and acrolein leading to cortical histological neuroprotection. J Neurotrauma 34: 1302-1317, 2017.

29. Jaganjac M, Tirosh O, Cohen G, Sasson S and Zarkovic N: Reactive aldehydes-second messengers of free radicals in diabetes mellitus. Free Radic Res 47 (Suppl 1): S39-S48, 2013.

30. Hall ED, Braughler JM, Yonkers PA, Smith SL, Linseman KL, Means ED, Scherch HM, Von Voigtlander PF, Lahti RA and Jacobsen EJ: U-78517F: A potent inhibitor of lipid peroxidation with activity in experimental brain injury and ischemia. J Pharmacol Exp Ther 258: 688-694, 1991.

31. Raza H and John A: 4-hydroxynonenal induces mitochondrial oxidative stress, apoptosis and expression of glutathione S-transferase A4-4 and cytochrome P450 2E1 in PC12 cells. Toxicol Appl Pharmacol 216: 309-318, 2006.

32. Grimsrud PA, Xie H, Griffin TJ and Bernlohr DA: Oxidative stress and covalent modification of protein with bioactive aldehydes. J Biol Chem 283: 21837-21841, 2008.

33. Schieber M and Chandel NS: ROS function in redox signaling and oxidative stress. Curr Biol 24: R453-R462, 2014.

34. Usatyuk PV and Natarajan V: Role of mitogen-activated protein kinases in 4-hydroxy-2-nonenal-induced actin remodeling and barrier function in endothelial cells. J Biol Chem 279: 11789-11797, 2004.

35. Huang Y, Li W and Kong AN: Anti-oxidative stress regulator NF-E2-related factor 2 mediates the adaptive induction of antioxidant and detoxifying enzymes by lipid peroxidation metabolite 4-hydroxynonenal. Cell Biosci 2: 40, 2012. 